\title{
Bifurcation Analysis of the Henon Map
}

\author{
ZHANYBAI T. ZHUSUBALIYEV ${ }^{\mathrm{a}, *}$, VADIM N. RUDAKOV ${ }^{\mathrm{a}}$, \\ EVGENIY A. SOUKHOTERIN ${ }^{a}$ and ERIK MOSEKILDE ${ }^{b}$

\begin{abstract}
${ }^{a}$ Kursk State Technical University, Department of Computer Science, 50 Years of October Street, 94, 305040 Kursk, Russia; ${ }^{b}$ Center for Chaos and Turbulence Studies, Department of Physics, Technical University of Denmark, 2800 Lyngby, Denmark
\end{abstract}

(Received 15 March 2000)

\begin{abstract}
Division of the parameter plane for the two-dimensional Hénon mapping into domains of periodic and chaotic oscillations is studied numerically and analytically. Regularities in the occurrence of different motions and transitions are analyzed. It is shown that there are domains in the plane of parameters, where non-uniqueness of motions exists. This may lead to abrupt changes of the character of the dynamics under variation in the parameters, that is, to a sudden transition from one stable cycle to another or to chaotization of the oscillations.
\end{abstract}

Keywords: Hénon two-dimensional mapping; Chaos; Bifurcations; Branching pattern

\section{INTRODUCTION}

The purpose of this paper is to apply bifurcation analysis and continuation methods to study regularities in the occurrence of different motions in the two-dimensional Hénon map (Hénon, 1976; Hitzl and Zele, 1981):

$$
\begin{aligned}
X_{k} & =F\left(\dddot{x}_{k-1}\right) ; \quad k=1,2, \ldots ; \\
X & =\left(x_{1}, x_{2}\right)^{T} ; F=\left(f_{1}, f_{2}\right)^{T} ; \\
f_{1} & =1-\alpha x_{1}^{2}+x_{2} ; \\
f_{2} & =\beta x_{1} .
\end{aligned}
$$

*Corresponding author.
Here $\alpha$ is the nonlinearity coefficient and $\beta$ is the coefficient of dissipation $(|\beta|<1) . T$ denotes the operation of transposition.

The mapping (1) was originally proposed by Hénon (Hénon, 1976) as a model of the Poincaré map for a three-dimensional flow system. Since then it has been extensively studied both numerically and from a more theoretical point of view (for references see, e.g., Schuster, 1984; Bergé et al., 1984; Moon, 1987; Anishchenko, 1990; Landa, 1996). Curry (Curry, 1979) was the first to observe that there exists a range of parameters for which two chaotic attractors coexist, and Derrida et al. (Derrida et al., 1979) showed how the 
period-doubling cascade in the Hénon map for certain parameter values follows the Feigenbaum scenario. At the same time, Newhouse (Newhouse, 1974) found that there are regions in parameter space in which a dense set of the stable periodic orbits with extremely small basins of attraction may exist. For a more recent discussion of this phenomenon see, e.g., Kan et al. (Kan et al., 1995). A variety of different motions generated by the two-dimensional Hénon map (Simo, 1979; Hansen and Cvitanović, 1998) in dependence of the parameters and peculiarities in the occurrence of chaos are typical of a wide class of dynamical systems (Schuster, 1984; Moon, 1987; Anishchenko, 1990; Mosekilde, 1996). As shown, for instance, by Anishchenko (Anishchenko, 1990) the map (1) is a good representation of a three-dimensional model of a radiophysical generator with inertial nonlinearity. In particular, it is shown that the collection of bifurcations in the plane of the controlling parameters $\alpha$ and $\beta$ is qualitatively equivalent to that which is realized in the Poincare map of a model of the generator. We have found a similar equivalence when analyzing models of a specific class of automatic control systems, for example, two- and three-dimensional models of systems with pulse-width modulation and a four-dimensional model of a relay system with hysteresis (Baushev and Zhusubaliyev, 1992; Baushev et al., 1996; Zhusubaliyev, 1997; Zhusubaliyev, 1997a).

Many other non-linear dynamical systems display a structure of division of the parameter plane and a shape and arrangement of the domains of stability similar to what we shall discuss here. The peculiarities of organization of domains of stability with the specific shape, referred to as a swallow tail, or a crossroad area and denoted here as $\Pi_{k}^{\text {Swallow }}$ can be observed with bimodal one-dimensional maps as shown in (Gallas, 1994), for the Ikeda map (Mosekilde, 1996), and for several other nonlinear dynamical systems (Barfred et al., 1996). Detailed analysis of such a structure have been performed by Kuznetsov et al. (Kuznetsov et al., 1993) and, from a more mathematical point of view, by Mira et al. (Mira et al., 1996) (see also references in this work). It is shown in (Kuznetsov et al., 1994), that a quantitative universality in the transition to chaos in two-dimensional Hénon-like maps exists. Hansen and Cvitanović (Hansen and Cvitanović, 1998) describe the possible bifurcation structures for maps of the Hénon type, in particular some swallow tail for various periods using a series of $n$-unimodal approximations and the associated symbolic dynamics. Sonis (Sonis, 1996) provides a detailed description of the bifurcation phenomena in the Hénon map.

Nonetheless, the complete bifurcation scenario for the Hénon map has not yet been established, and the aim of the present article is to present a number of results that appear not to have been given in the above works. Special emphasis is paid to the study of the structure and properties of the division of the plane of controlling parameters for the dynamical system (1) into the domains of existence of periodic motions and chaos.

\section{DIVISION OF PARAMETER PLANE}

Let us begin with some preliminary notes.

Let $X_{c i}, i=\overline{1, m}$ be a periodic motion ( $m$-cycle) of the dynamic system (1). It is evident that all $X_{c i}$, $i=\overline{1, m}$ satisfy the following equation

$$
X_{c}-F^{m}\left(X_{c}\right)=0, F^{m}\left(X_{c}\right)=\underbrace{F\left(F\left(\ldots\left(F\left(X_{c}\right)\right) \ldots\right)\right)}_{m \text { times }} .
$$

The local stability of the $m$-cycle is determined by the eigenvalues (multiplicators) $\rho_{1}$ and $\rho_{2}$ of the basic matrix $\Phi_{m}$. These are the roots of the equation

$$
\begin{gathered}
\operatorname{det}\left(\Phi_{m}-\rho E\right)=0, \\
\Phi_{i}=\frac{\partial F\left(X_{c(i-1)}\right)}{\partial X_{c(i-1)}} \Phi_{i-1}, \Phi_{0}=E, i=\overline{1, m} \\
\frac{\partial F\left(X_{c(i-1)}\right)}{\partial X_{c(i-1)}}=\left|\begin{array}{cc}
-2 \alpha X_{1 c(i-1)} & 1 \\
\beta & 0
\end{array}\right|
\end{gathered}
$$


Here $E$ is the unit matrix.

Let $\Pi=\{(\alpha, \beta): \mu<\alpha<\nu ; \vartheta<\beta<\gamma\}$ be the set of the parameters of the dynamic system (1). A point in the plane $P=(\alpha, \beta) \in \Pi$ corresponds to every fixed set of parameter values. Let $X_{c i}(P), i=$ $\overline{1, m}$ be a locally stable $m$-cycle corresponding to point $P$.

Let $\Pi_{k, j}$ be the simply connected set of parameters $\Pi_{k, j} \subset \Pi, k \geq 1 ; j=1,2, \ldots, s$ such, that for every $P \in \Pi_{k, j}$ there exists a stable $m$-cycle $X_{c i}(P)$, $i=\overline{1, m}$, which is continuous along the parameters in $\Pi_{k, j}$. If there are several motions at fixed $P$, different $\Pi_{k, j}$ correspond to different motions. The $k$-cycle for $\Pi_{k, j}$ is minimum, that is, there is no $m$ cycles with $m<k$ in $\Pi_{k, j}$. The index $j$ is introduced to distinguish sets, having the same $k$. The value of $s$ may be finite or infinite.

The boundaries of the sets $\Pi_{k, j}$ may be of the following types:

- simple, that is, there is a stable $m$-cycle for any points $P \in \Gamma_{k, j}$, connected with a limitation of the parameter variation range;

- the boundaries $\Gamma_{k, j}$ are formed by the collection of bifurcation values of parameters or by the collection of points of accumulation, corresponding to aperiodic motions.

The point $P_{*} \in \Pi$ is a bifurcation point, if the equation (Neymark and Landa, 1987; Butenin et al., 1987)

$$
\begin{aligned}
& \chi(P, \rho)=\rho^{2}+\xi_{1} \rho+\xi_{2}=0, \\
& \chi(P, \rho)=\operatorname{det}\left(\Phi_{m}-\rho E\right)
\end{aligned}
$$

has a root, which is on the unit circle, when $P=P_{*}$.

These are the cases, when the greatest multiplicator of $m$-cycle (in absolute value) turns into 1 or -1 . The corresponding bifurcation curves are determined by the equations

$$
\begin{aligned}
\chi(P, 1) & =1+\xi_{1}+\xi_{2}=0 \\
\chi(P,-1) & =1-\xi_{1}+\xi_{2}=0
\end{aligned}
$$

By analogy with (Neymark and Landa, 1987; Butenin et al., 1987) here and in the following we denote these curves as $N_{+}$and $N_{-}$, respectively. For the dissipative system (1), the case where two complex conjugate eigenvalues cross the unit circle cannot occur.

It is interesting to study the possibility of nonempty intersections of the sets $\Pi_{k, j}$ and the properties of $\Pi_{k, j}$ in whole in order to understand the great variety of different motions, generated by the dynamical system (1). In the following we shall restrain ourselves to the region $\Pi=\{(\alpha, \beta)$ : $-1<\alpha<4 ;|\beta|<1\}$.

Let us first consider the solutions of Eq. (2) and study the local stability of $m$-cycles.

Equation (2) when $m=1$ has the form:

$$
\begin{aligned}
& \alpha x_{1 c}^{2}+(1-\beta) x_{1 c}-1=0 \\
& x_{2 c}=\beta x_{1 c} .
\end{aligned}
$$

The roots of this equation determine two period-1 cycles

$$
\begin{aligned}
& x_{1 c}=\frac{1}{2 \alpha}\left[\beta-1 \pm \sqrt{(1-\beta)^{2}+4 \alpha}\right] \\
& x_{2 c}=\beta x_{1 c} .
\end{aligned}
$$

The domain of existence of a stable 1-cycle is limited by bifurcation curves $N_{+}, N_{-}$:

$$
\beta^{2}-2 \beta+4 \alpha+1=0
$$

and

$$
3 \beta^{2}-6 \beta-4 \alpha+3=0,
$$

respectively. Let us denote this domain as $\hat{\Pi}_{1,1}$ :

$\widehat{\Pi}_{1,1}=\left\{(\alpha, \beta):-\frac{1}{4}(\beta-1)^{2} \leq \alpha \leq \frac{3}{4}(\beta-1)^{2},|\beta|<1\right\}$.

If the parameters $P \in \tilde{\Pi}_{1}, \quad \tilde{\Pi}_{1}=\{(\alpha, \beta):-1$ $\left.<\alpha<-(1 / 4)(\beta-1)^{2},|\beta|<1\right\}$, then Eq. (3) has no real roots. On the curve $\alpha=-(1 / 4)(\beta-1)^{2}$, $-1<\beta<1$ Eq. (3) takes the form

$$
\left(x_{1 c}-\frac{2}{1-\beta}\right)^{2}=0
$$


and has a multiple root. When changing the parameters along some smooth curve (referred to as the trajectory of deformation) into the domain $\alpha>-(1 / 4)(\beta-1)^{2}, \quad-1<\beta<1$, two solutions arise: one of which corresponds to a stable 1-cycle and the other to an unstable 1-cycle. This pair of 1 -cycles occurs abruptly in a saddle-node bifurcation at $\alpha=-(1 / 4)(\beta-1)^{2}$. In a reverse transition, the stable 1-cycle disappears as it coincides with the unstable cycle at the points of intersection with the trajectory of deformation and curve (4).

It is easy to see, that $\alpha=-(1 / 4)(\beta-1)^{2}<0$. Now let us follow the evolution of the stable and unstable 1-cycles with variation in $\alpha$ from negative values to positive ones by passing through $\alpha=0$. Denote the solutions of the Eq. (2) as $X_{c}^{S}$, $X_{c}^{U}$, that correspond to stable 1-cycle and unstable 1 -cycle.

When $\alpha=0$, Eq. (2) has only one solution

$$
x_{1 c}=\frac{1}{1-\beta}, \quad x_{2 c}=\frac{\beta}{1-\beta} .
$$

It is obvious, that

$$
\lim _{\alpha \rightarrow \pm 0} x_{1 c}^{S}=\frac{1}{1-\beta}, \quad \lim _{\alpha \rightarrow \pm 0} x_{2 c}^{S}=\frac{\beta}{1-\beta} .
$$

Hence the solution of Eq. (2), which corresponds to the stable 1-cycle, depends smoothly on the parameters in $\widehat{\Pi}_{1,1}$, whereas $X_{c}^{U}$ is uninterrupted in $\widehat{\Pi}_{1,1}$ except for the points, in which $\alpha=0$. In points $\alpha=0,|\beta|<1$ the solution $X_{c}^{U}$ has an infinite discontinuity.

The distinction in the nature of the parameter dependence of the solutions $X_{c}^{S}$ and $X_{c}^{U}$ is shown in Figure 1(a). The dependences of the multiplicators of stable and unstable 1 -cycles are given in Figure 1(b).

When the trajectory of deformation and the bifurcation curve (5) intersect, the 1-cycle loses its stability, and a stable 2-cycle arises softly as the result of a period-doubling bifurcation. Both 1cycles continue to exist as unstable over the whole range of parameters beyond bifurcation curve (5).

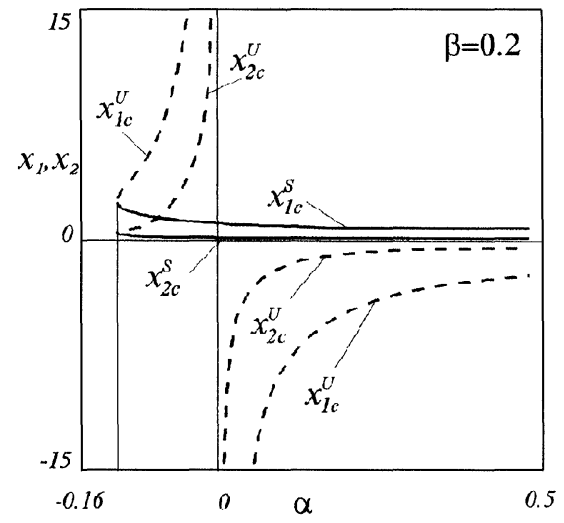

(a)

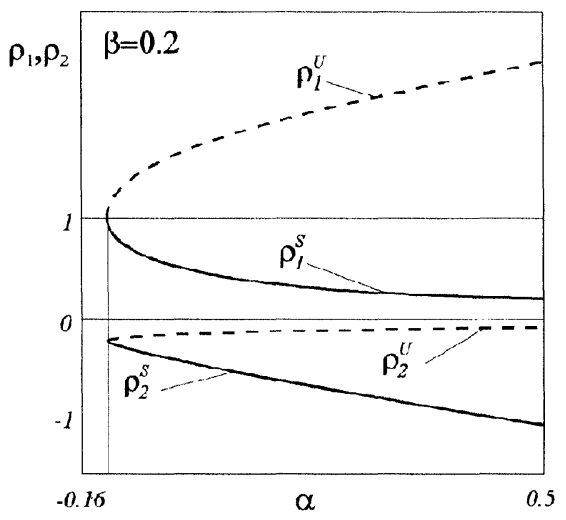

(b)

FIGURE 1 (a) Variation of the stable and unstable period-1 cycles with the nonlinearity parameter $\alpha$. Note the different nature of these dependences. (b) Corresponding variation of the multiplicators $\rho_{1}$ and $\rho_{2}$.

Equation (2) when $m=2$ takes the form

$$
\begin{aligned}
\alpha^{3} x_{1 c}^{4} & -2 \alpha^{2} x_{1 c}^{2}+(1-\beta)^{3} x_{1 c}-(1-\beta)^{2}+\alpha=0 \\
x_{2 c} & =\frac{\beta}{1-\beta}\left(1-\alpha x_{1 c}^{2}\right) .
\end{aligned}
$$

This equation has four real roots in the domain $\alpha>(3 / 4)(\beta-1)^{2}$. Two of the roots correspond to two unstable 1 -cycles. It is not difficult to show, that a stable 2-cycle satisfies the equation

$$
\begin{aligned}
& \alpha^{2} x_{1 c}^{2}-\alpha(1-\beta) x_{1 c}+(1-\beta)^{2}-\alpha=0 \\
& \quad x_{2 c}=\frac{\beta}{1-\beta}\left(1-\alpha x_{1 c}^{2}\right) .
\end{aligned}
$$


The set $\widehat{\Pi}_{2,1}$, whereupon a stable 2-cycle is determined, is limited by the bifurcation curve (5) and by the curve, defined by the following equation

$$
5 \beta^{2}-6 \beta-4 \alpha-5=0
$$

that is,

$$
\begin{aligned}
\widehat{\Pi}_{2,1}=\{ & (\alpha, \beta): \frac{3}{4}(\beta-1)^{2} \leq \alpha \\
& \left.\leq \frac{1}{4}\left(5 \beta^{2}-6 \beta-5\right),|\beta|<1\right\} .
\end{aligned}
$$

When passing through the boundary (7) into the domain of values $\alpha>(1 / 4)\left(5 \beta^{2}-6 \beta-5\right),|\beta|<1$, a stable 4-cycle arises softly, and the 2-cycle continues to exist, but becomes a saddle.

It becomes more and more difficult to obtain bifurcation formulas in explicit form for $m=2^{i-1}$, $i=3,4, \ldots$. All the rest of the sets $\widehat{\Pi}_{2^{i-1}, 1}, i=3,4, \ldots$ were built numerically. The collection of $\widehat{\Pi}_{2^{i-1}, 1}$, $i=1,2, \ldots$ which forms $\Pi_{1,1}$, is shown in Figure 2 :

$$
\Pi_{1,1}=\bigcup_{i=1}^{\infty} \widehat{\Pi}_{2^{i-1}, 1}
$$

In this way, the set $\Pi_{1,1}$ consists of domains of existence of stable $2^{i-1}$-cycles $(i=1,2,3, \ldots)$. The boundaries, separating these domains in $\Pi_{1,1}$ correspond to period-doubling bifurcation curves. The set $\Pi_{1,1}$ is limited by bifurcation curve (4) from the left and by a curve formed by the collection of accumulation points from the right.

Now consider $\Pi_{k, j}=\bigcup_{i=1}^{\infty} \Pi_{k \cdot 2^{i-1}, j}$ for $k \neq 1$. From Eq. (2) when $m=3$ we find

$$
\begin{aligned}
\alpha^{6} x_{1 c}^{6} & -\alpha^{5}(1-\beta) x_{1 c}^{5}+\alpha^{4}\left((1-\beta)^{2}-3 \alpha\right) x_{1 c}^{4}- \\
& -\alpha^{3}(1-\beta)\left(1+\beta^{2}-2 \alpha\right) x_{1 c}^{3}+ \\
& +\alpha^{2}\left(\beta^{4}-\beta^{2}+1-(3 \alpha+2 \cdot \beta) .\right. \\
& \left.\cdot\left(1-\alpha+\beta^{2}\right)\right) x_{1 c}^{2}+ \\
& +\alpha(\beta-1)\left(\left(1+\beta+\beta^{2}\right)^{2}+\alpha^{2}-2 \alpha\right) x_{1 c}+ \\
& +1+\beta+\beta^{2}+\beta^{4}+\beta^{5}+\beta^{6}- \\
& -\alpha\left(1-2 \alpha+\alpha^{2}-4 \beta-\right. \\
& \left.\quad-5 \beta^{2}-4 \beta^{3}+\beta^{4}-2 \alpha \beta^{2}\right)=0 .
\end{aligned}
$$

The roots of this equation can be found only numerically. Let us try to find the equation of bifurcation curve $N_{+}$for the 3-cycle. Taking into account, that with parameter values, located on the curve $N_{+}$, Eq. (8) has only multiple roots, we

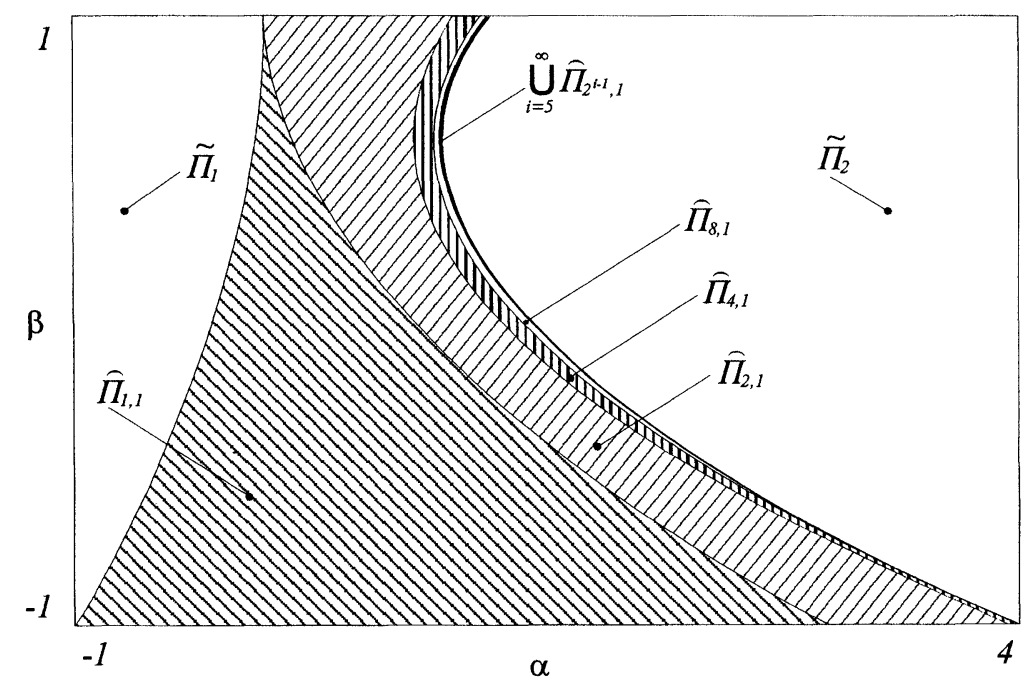

FIGURE 2 The collection of the sets $\hat{\Pi}_{2^{i-1}, 1}, i=1,2, \ldots$ which forms $\Pi_{1,1}, \tilde{\Pi}_{1}$ and $\tilde{\Pi}_{2}$ denote those domains of $\Pi$, where trajectories diverge to infinity with any initial conditions. 
obtain

$$
x_{1 c}^{3}-\frac{1-\beta}{2 \alpha} x_{1 c}^{2}+\sigma x+\vartheta=0 .
$$

Here

$$
\begin{gathered}
\sigma=\frac{1}{2 \sqrt{\vartheta}}\left(2 \alpha-\alpha^{2}-1-\beta-\beta^{2}+\beta^{3}\right. \\
\left.+\beta^{4}+\beta^{5}+\alpha^{2} \beta-2 \alpha \beta^{3}\right)
\end{gathered}
$$

where

$$
\begin{aligned}
\vartheta & =1+\beta+\beta^{2}+\beta^{4}+\beta^{5}+\beta^{6} \\
& -\alpha\left(1-2 \alpha+\alpha^{2}-4 \beta-5 \beta^{2}-4 \beta^{3}+\beta^{4}-2 \alpha \beta^{2}\right) .
\end{aligned}
$$

Then the desired bifurcation curve will lie on the two-dimensional surface (Fig. 3(a))

$$
\begin{aligned}
\chi(\alpha, \beta, 1)= & -8[\mu(\alpha, \beta)]^{(1 / 2)}+(1-\beta)(1+\beta)^{2}, \\
\mu(\alpha, \beta)= & 1+\beta+\beta^{2}+\beta^{4}+\beta^{5}+\beta^{6} \\
& -\alpha\left(1+\alpha^{2}-4 \beta-5 \beta^{2}-4 \beta^{3}+\beta^{4}\right) \\
& +2 \alpha^{2}\left(1+\beta^{2}\right) .
\end{aligned}
$$

We find an equation for the bifurcation curve, by setting $\chi(\alpha, \beta, 1)$ equal to zero:

$$
-8[\mu(\alpha, \beta)]^{(1 / 2)}+(1-\beta)(1+\beta)^{2}=0 .
$$

The form of this curve is shown in Figure 3(b). The remaining domains $\widehat{\Pi}_{3 \cdot 2^{i-1}, 1}, i=2,3, \ldots$, as well as $\widehat{\Pi}_{2^{i-1}, 1}, i=3,4, \ldots$, were built numerically.

Figure 4 shows the collection

$$
\begin{gathered}
\Pi_{3,1}=\bigcup_{i=1}^{\infty} \widehat{\Pi}_{3 \cdot 2^{i-1}, 1} ; \Pi_{k, 1}=\bigcup_{i=1}^{\infty} \widehat{\Pi}_{k \cdot 2^{i-1}, 1}, \\
k=5,6,7,8,10,12
\end{gathered}
$$

and

$$
\Pi_{k, 2}=\bigcup_{i=1}^{\infty} \widehat{\Pi}_{k \cdot 2^{i-1}, 2}, \quad k=5,6,8
$$

which were obtained numerically. Naturally, Figure 4 represents only those sets, that are relatively large in $\Pi$.

The resulting picture for the division of Hénon mapping parameter plane $\Pi=\{(\alpha, \beta):-1<\alpha<4$; $|\beta|<1\}$ into domains of various oscillatory modes, is shown in Figure 5. The domains of chaoticity adjoin to the parts of boundaries of $\Pi_{k, j}$, formed by the set of accumulation points. The diagram depicts only one of them, which occupies the largest region in the parameter plane. The domain is shown in Figures 4, 5 by dark hatching and is denoted as $\Pi_{\text {Chaos }}$. The remaining domains are very narrow and hence, are not shown in the diagram. There is a great number of windows with deterministic dynamics within $\Pi_{\text {Chaos, }}$ which begin with saddle-node branching $m$-cycles. The inner structure of such domains may be similar to that for $\Pi_{k, j}$ or be different. The domains, whose properties are different from the properties of $\Pi_{k, j}$, are denoted as $\Pi_{k}^{\text {Swallow }}$ (see Fig. 4). The structure of $\Pi_{k}^{\text {Swallow }}$ and the bifurcations in this domain were described in some detail in (Dmitriev et al., 1994).

For any point $P \in \Pi$ with the exception of $P=(0,0)$ there is such (simply or not simply connected) domain $D_{*}$ in the phase space of the dynamic system (1), that if $X_{0} \in D_{*}$, then

$$
\lim _{k \rightarrow \infty}\left\|X_{k}\left(X_{0}\right)\right\|=\infty, \quad X_{k}\left(X_{0}\right)=F^{k}\left(X_{0}\right) .
$$

In Figure 5 ( $c f$. also Fig. 2) $\tilde{\Pi}_{1}$ and $\tilde{\Pi}_{2}$ denote those domains of $\Pi$ (not hatched), where Eq. (9) with any $X_{0}$ is valid for all points $P \in \tilde{\Pi}_{1} \cup \tilde{\Pi}_{2}$.

As seen in Figures 4, 5, all sets $\Pi_{k, j}$, with the exception of $\Pi_{k}^{\text {Swallow }}$, have non-empty intersections with $\Pi_{1,1}$. Moreover $\Pi_{i, l} \cap \Pi_{q, p} \neq \emptyset(i, q \neq 1$, $i \neq q)$. This means, that there exist different stable $m$-cycles, corresponding to $\Pi_{i, l}$ and $\Pi_{q, p}$ for every $P \in \Pi_{i, l} \cap \Pi_{q, p}$.

It is easy to see, that

$$
\begin{gathered}
\Pi_{3,1} \cap \Pi_{5}^{\text {Swallow }} \neq \phi, \Pi_{i}^{\text {Swallow }} \\
\cap \Pi_{j}^{\text {Swallow }} \neq \phi, \quad i \neq j .
\end{gathered}
$$




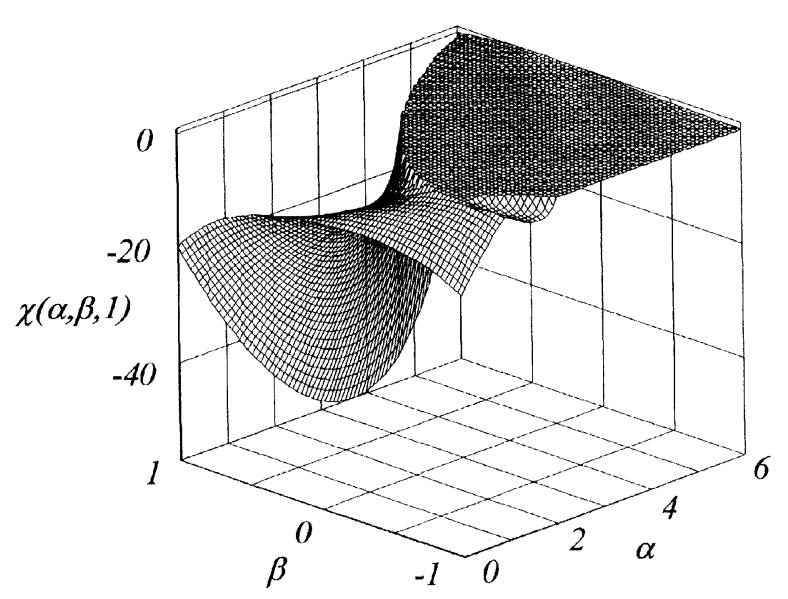

(a)

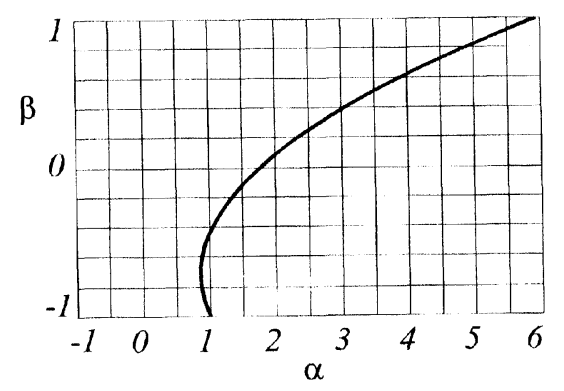

(b)

FIGURE 3 (a) The 2D surface, on which the bifurcational curve $N_{+}$for 3-cycle lies. (b) The curve of saddle-node bifurcation for 3-cycle.

Based on these results let us elaborate on the bifurcations analysis, while moving along the parameters in the domain $\left(\bigcup_{(k, j)} \Pi_{k, j}\right) \cup \Pi_{\text {Chaos }}$ in more detail.

The analysis was made in the sections $\beta=0.3$; $\beta=0.68$ and $\beta=0.5$, changing the parameter $\alpha$ over the range of

$$
\begin{aligned}
\Omega_{\alpha}=\{\alpha & :-\frac{1}{4}(\beta-1)^{2} \\
& <\alpha<\nu,|\beta|<1, \nu<4,(\nu, \beta=\text { const })\} .
\end{aligned}
$$

The bifurcation diagrams calculated for these values of $\beta$ and the indicated variation of $\alpha$ are depicted in Figures 6(a), 7(a) and 8. It is easy to see in these diagrams, that the complication of oscillations happens through a sequence of period-doubling bifurcations while continuously increasing $\alpha$. These sequences lead to the appearance of aperiodic motion with some critical value of $\alpha$ and then to the domain of chaoticity. The ranges of variation in $\alpha$ in which chaotic oscillations are observed, are interrupted by small intervals, where stable cycles exist (Figs. $6-8$ ).

In the domain $-(1 / 4)(\beta-1)^{2}<\alpha<\nu,|\beta|<1$, $\nu<4,(\nu, \beta=$ const $)$ with variation in $\alpha$ many other stable motions occur in saddle-node bifurcations, such as 3-, 6-, 8-, 18-cycles and other motions with 


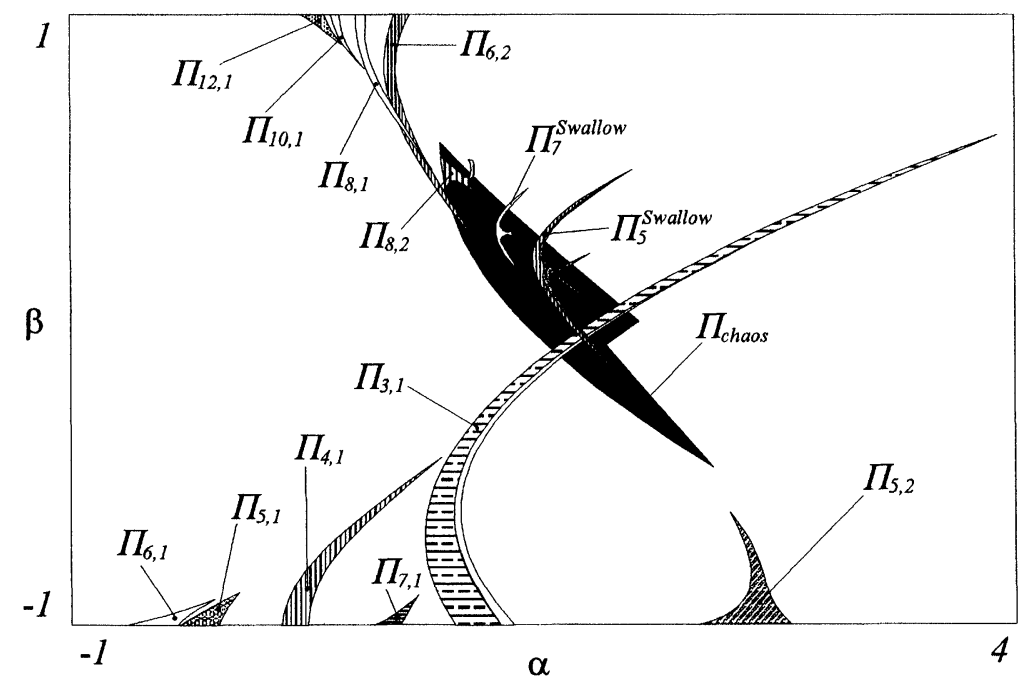

FIGURE 4 The collections of the sets $\Pi_{3,1}, \Pi_{k, 2^{i-1}, 1}, i=1,2, \ldots, k=5,6,7,8,10,12$ and $\Pi_{k, 2}, k-5,6,8$. Here $\Pi_{\text {Chaos }}$ is the domain the chaoticity.

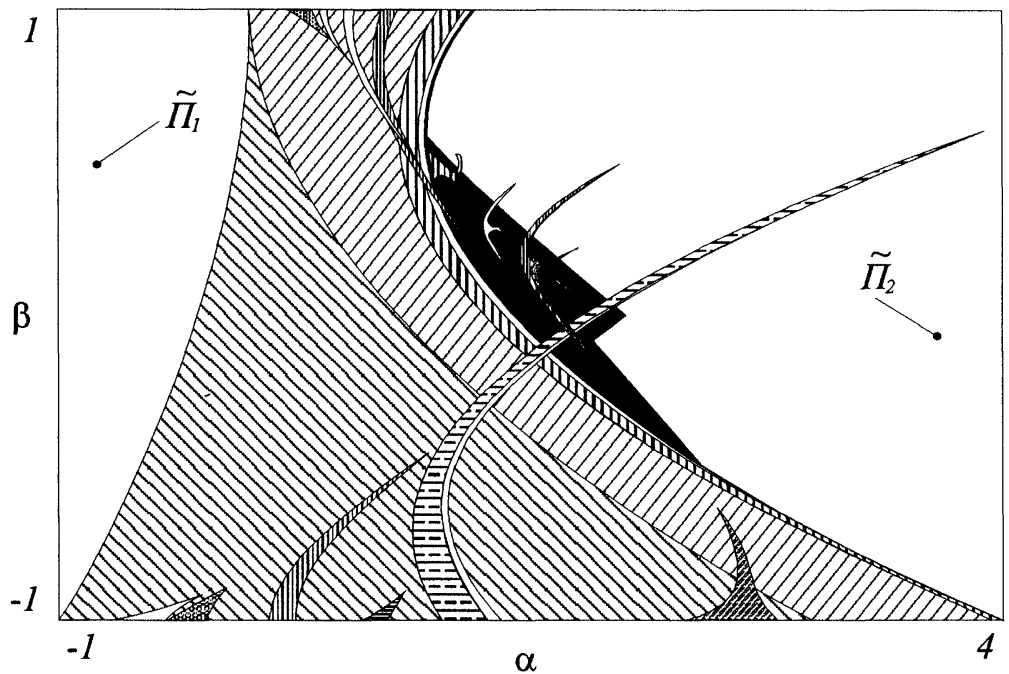

FIGURE 5 Resulting picture for the division of Hénon map parameter plane into domains of various oscillatory modes.

subsequent period-doubling bifurcations. That is why the general pattern of cycles branching is complicated significantly.

Let us follow the evolution of various stable cycles, with variation in $\alpha$. The results of numerical calculations for $\beta=0.3$ and $\beta=0.68$ are presented in Tables I, II. In these tables the second column contains the periodicity of a cycle, the forth and the fifth columns are the range of $\alpha$, in which locally stable $m$-cycle exists. The last column indicates the width of this range.

It is convenient to represent the data of the tables in the form of a diagram, which was called branching pattern (Figs. 6(b) and 7(b)) in 


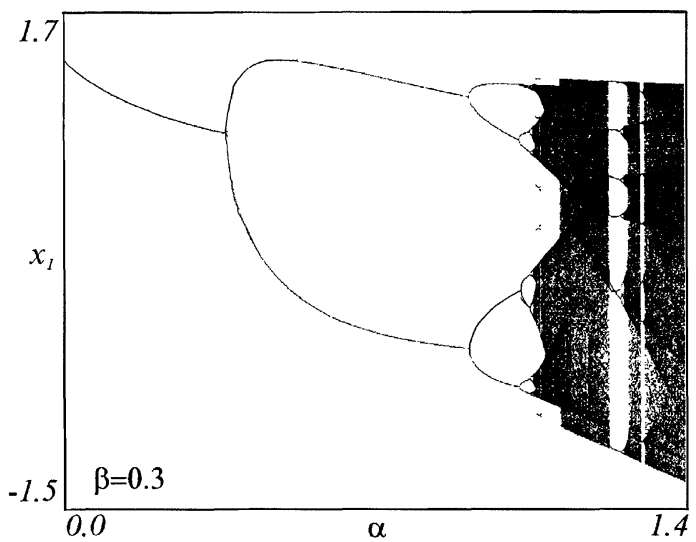

(a)

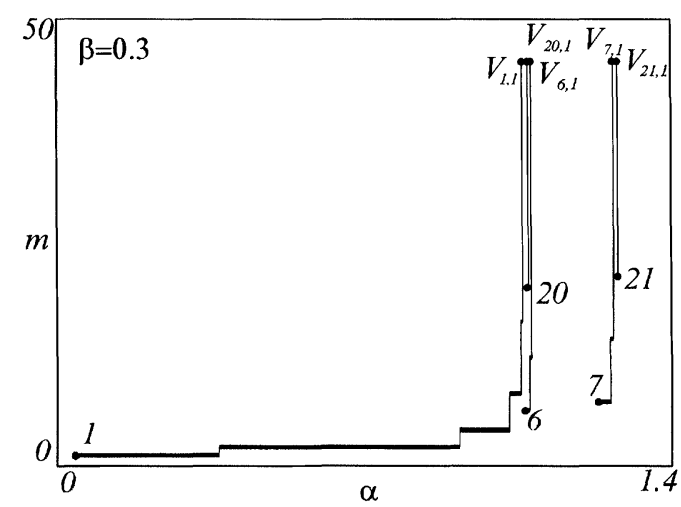

(b)

FIGURE 6 (a) Bifurcation diagram and (b) branching pattern for $0 \leq \alpha \leq 1.4$ and $\beta=0.3$.

(Baushev and Zhusubaliyev, 1992; Baushev et al., 1996). In these diagrams values of varying parameters are plotted on the horizontal axis and values of $m$ are plotted on the vertical axis. Further, we use designations as in (Baushev et al., 1996). $V_{k, j}$ are the branches, where index $k$ indicates from which $k$-cycle the branch begins. The maximum value $j$ indicates the number of observed branches, having the same $k$. All branches start with $k$-cycles, which arise in a saddle-node bifurcations. Some $V_{k, j}$ are determined on sets, that were not shown in Figure 5, as their sizes in parameter plane are too small.

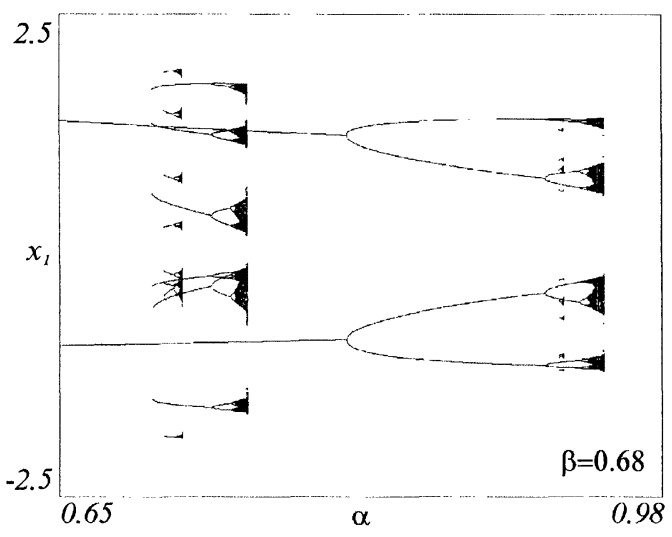

(a)

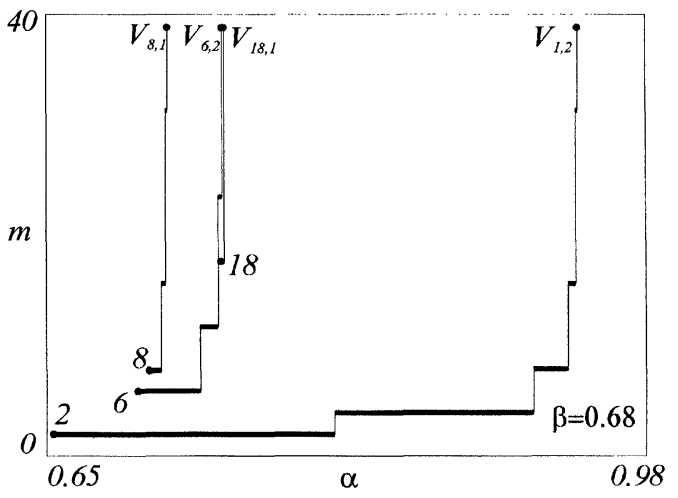

(b)

FIGURE 7 (a) Bifurcation diagram and (b) branching pattern for $0.65 \leq \alpha \leq 0.98$ and $\beta-0.68$.

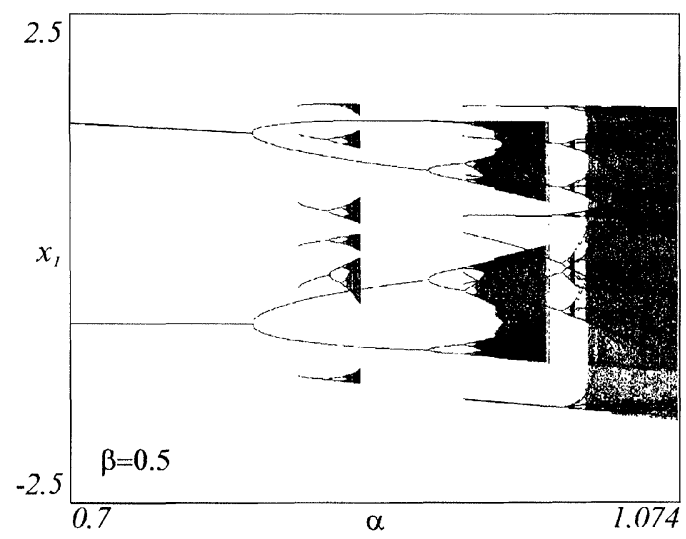

FIGURE 8 Bifurcation diagram for $0.7 \leq \alpha \leq 1.074$ and $\beta=0.5$. 
TABLE I

\begin{tabular}{llllll}
\hline$V_{k, j}$ & $m$ & $i$ & \multicolumn{1}{c}{$\alpha_{2 \cdot k, j}^{i-1}$} & \multicolumn{1}{c}{$\alpha_{2 \cdot k, j}^{i}$} & $\Delta \alpha$ \\
\hline$V_{1,1}$ & 1 & 1 & -0.1225 & 0.3675 & 0.49 \\
& 2 & 2 & 0.3675 & 1.0125 & 0.545 \\
& 4 & 3 & 0.9125 & 1.0511256620352 & 0.1133554050738 \\
& 8 & 4 & 1.0258554050738 & 1.0565637581588 & 0.0252702569614 \\
& 16 & 5 & 1.0511256620352 & 1.0577304656609 & 0.00116670750961236 \\
$V_{6,1}$ & 32 & 6 & 1.0565637581588 & 1.057956 & 0.0002255343391 \\
& 64 & 7 & 1.0577304656609 & 1.0710703629071 & 0.0086983629071 \\
$V_{7,1}$ & 6 & 1 & 1.062372 & 1.0750124047736 & 0.0039420418665 \\
& 12 & 2 & 1.0710703629071 & 1.2541834642429 & 0.0275660642429 \\
& 7 & 1 & 1.2266174 & 1.2600151726701 & 0.0058317084272 \\
$V_{20,1}$ & 14 & 2 & 1.2541834642429 & 1.2614289068416 & 0.0014137341715 \\
$V_{21,1}$ & 28 & 3 & 1.2600151726701 & 1.2617 & 0.0002710931584 \\
& 56 & 4 & 1.2614289068416 & 1.0677430954543 & 0.0001790954543 \\
\end{tabular}

TABLE II

\begin{tabular}{ccllll}
\hline$V_{k, j}$ & $m$ & $i$ & \multicolumn{1}{c}{$\alpha_{2 \cdot k, j}^{i-1}$} & \multicolumn{1}{c}{$\alpha_{2 \cdot k, j}^{i}$} & $\Delta \alpha$ \\
\hline$V_{1,2}$ & 2 & 2 & 0.0768 & 0.808 & 0.7312 \\
& 4 & 3 & 0.808 & 0.9169381427467 & 0.1089381427467 \\
& 8 & 4 & 0.9169381427467 & 0.9361479503607 & 0.019209807614 \\
& 16 & 5 & 0.9361479503607 & 0.9400330556502 & 0.0038851052895 \\
& 32 & 6 & 0.9400330556502 & 0.9408680756308 & 0.0008350199806 \\
$V_{6,2}$ & 64 & 1 & 0.9408680756308 & 0.9410471449543 & 0.0001790693235 \\
& 6 & 2 & 0.70142261 & 0.7343659105724 & 0.0329433005724 \\
& 12 & 3 & 0.7343659105724 & 0.7445017603884 & 0.010135849816 \\
$V_{8,1}$ & 24 & 4 & 0.7467731395103 & 0.7467731395103 & 0.0022713791219 \\
& 48 & 1 & 0.70791248 & 0.7472652867434 & 0.0004921472331 \\
& 16 & 2 & 0.713479366256 & 0.713479366256 & 0.005566886256 \\
& 32 & 3 & 0.7158180118385 & 0.7158180118385 & 0.0023386455825 \\
$V_{18,1}$ & 64 & 4 & 0.7163564950326 & 0.7163564950326 & 0.0005384831941 \\
& 18 & 1 & 0.74696954 & 0.7476245768696 & 0.000117446971 \\
& 36 & 2 & 0.7476245768696 & 0.7479207404767 & 0.0006550368696 \\
\end{tabular}

The range of existence of each branch $V_{k, j}$ is presented in the form of intervals

$$
\alpha_{k, j}<\alpha_{2 \cdot k, j}<\cdots<\alpha_{2^{i} \cdot k, j}<\cdots<\alpha_{k, j^{*}} .
$$

Here the parameters $\alpha_{2^{i} k, j}, i=1,2, \ldots$ correspond to loss of stability of the $2^{i-1} k$-cycle and soft occurrence of the $2^{i} k$-cycle. When $\mathbf{i}$ increases, the intervals $\Delta \alpha_{2^{i} k, j}=\alpha_{2^{i+1} k, j}-\alpha_{2^{i} k, j}$ of the existence of the $2^{i} k$-cycle become narrower (see Tabs. I, II), so that each branch has its limit value of $\alpha_{k, j^{*}}$, which corresponds to aperiodic motion. We shall call these parameters accumulation points as in (Baushev et al., 1996). The accumulation points are shown in Figures 6(b) and 7(b) as vertical lines. The beginning of the windows of periodicity in the bifurcation diagrams coincides with the beginning of the branches. Some of $V_{k, j}(k \neq 1)$ intersect with $V_{1,1}$. In those ranges of $\alpha$, where $V_{1,1} \cup V_{k, j} \neq \phi$, $k \neq 1$, there exist both a stable $2^{i-1}$-cycle and stable $2^{d-1} k$-cycle, $k \geq 3 ; i, d \geq 1$. This means, that the phase plane $\left(x_{1}, x_{2}\right)$ of the dynamic system (1) comprises some basins of attraction of different cycles. In this case the $2^{i-1}$-cycle or the $2^{d-1} k$ cycle are chosen, depending on the initial 
conditions. The character of division of the phase plane of system (1) into basins of attraction of different cycles is shown in Figures 9-11. Figures 9(a) and (b) display basins of attraction for the 1 -cycle and the 3 -cycle $(P=(0.949 ;-0.744))$.
In Figure 10 we show basins of attraction for the 1-cycle, the 18-cycle, and the 3-cycle, $(P=(1.0295 ;-0.8))$, and in Figure 11 are basins of attraction for the 2-cycle and the 8-cycle $(P=(0.53 ; 0.88))$.

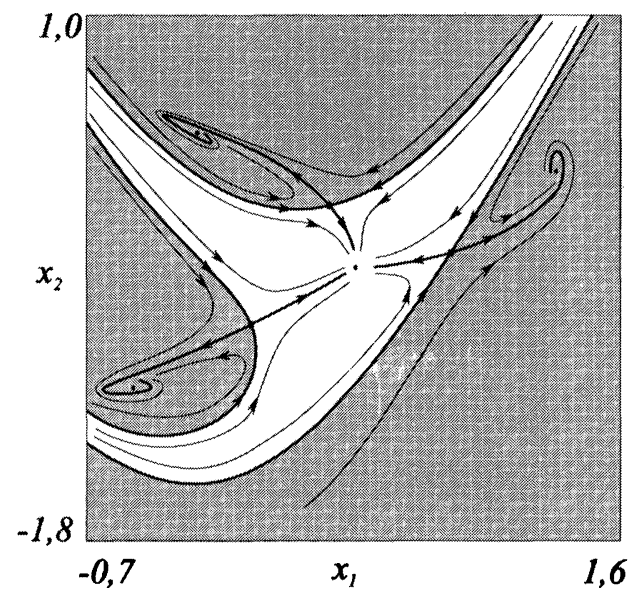

(a)

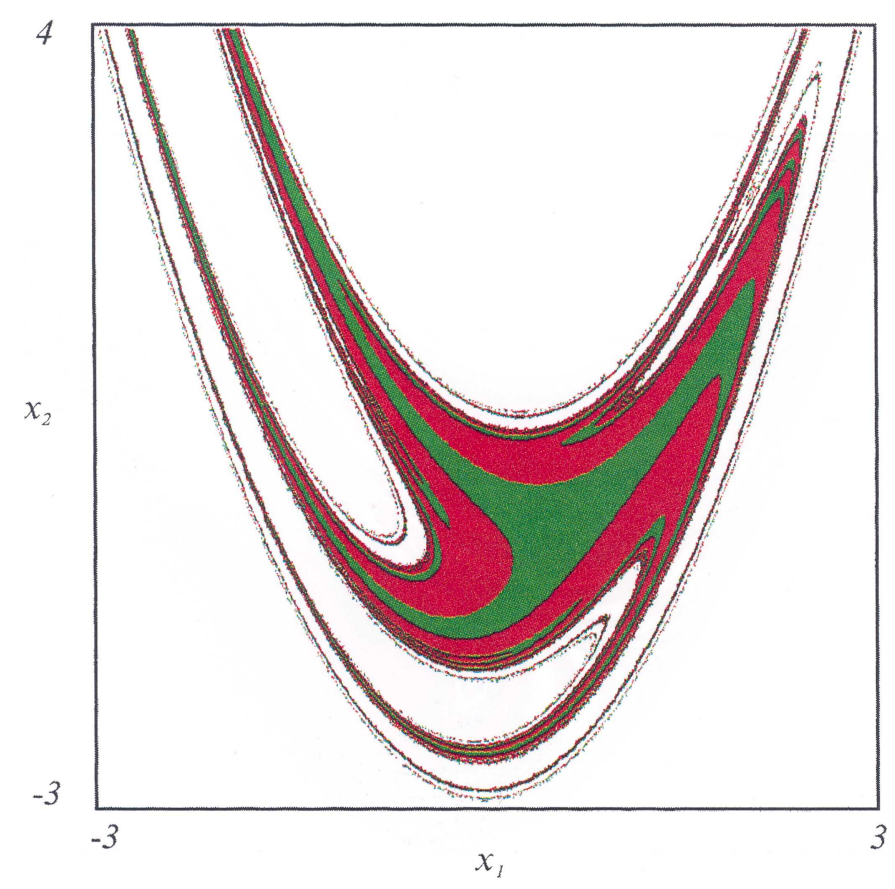

FIGURE 9 (a), (b) Basins of attraction for the 1-cycle and the 3-cycle with $\alpha=0.949, \beta=-0.744$. (See Color Plate II.) 


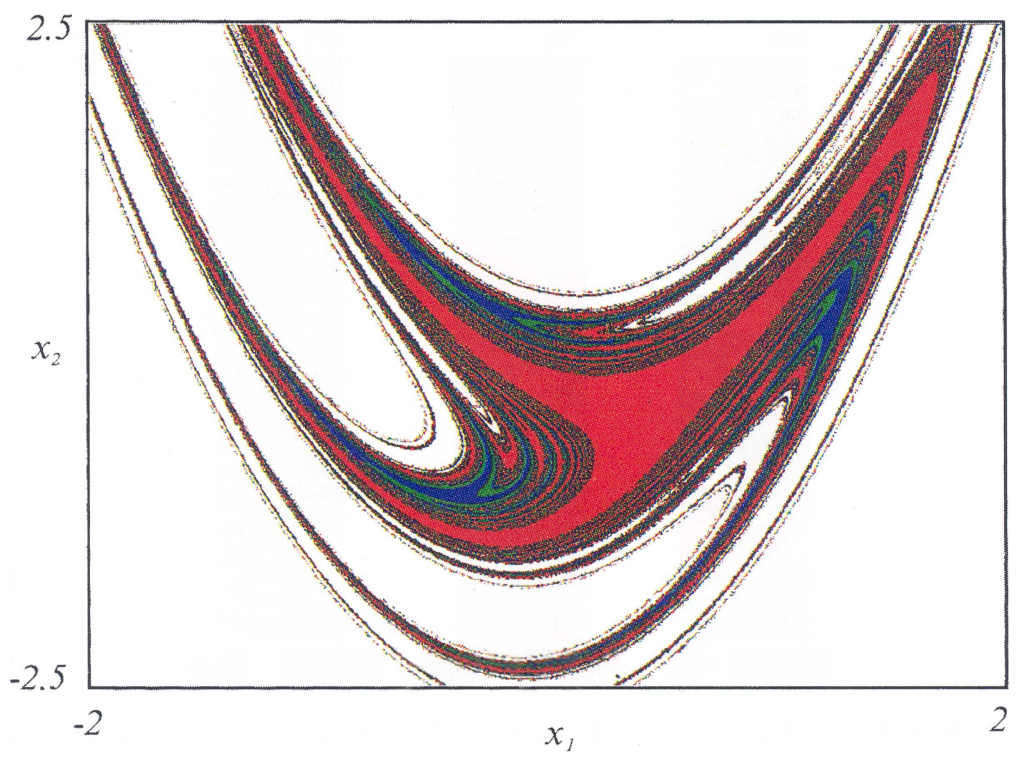

FIGURE 10 Basins of attraction for the 1-cycle, the 18-cycle, and the 3 -cycle with $\alpha=1.0295, \beta=-0.8$. (See Color Plate III.)

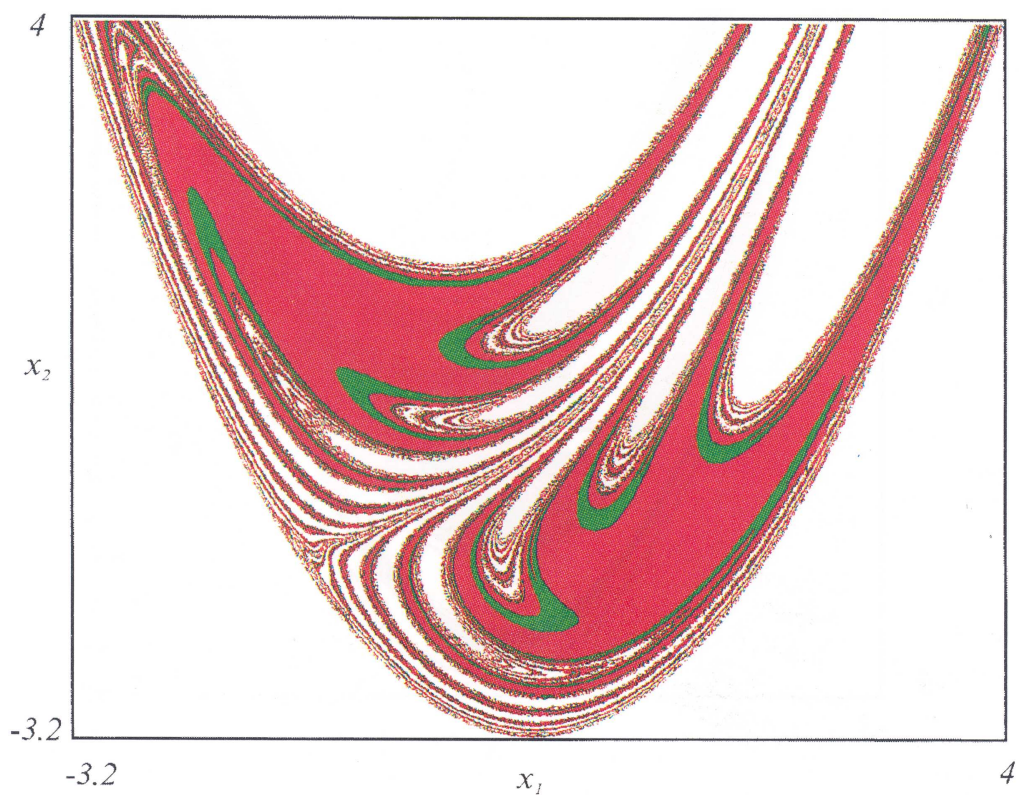

FIGURE 11 Basins of attraction for the 2-cycle and the 8-cycle with $\alpha=0.53, \beta=0.88$. (See Color Plate IV.)

The location of $X_{c k}, k=\overline{1, m}$, are marked by dots in Figures 9-11. The non-hatched parts of Figures 9 and 10 correspond to the collection of initial conditions from which the trajectories diverge to infinity (see (9)).

One can see from the comparison of the branching pattern with the bifurcation diagrams that there are intervals within the limits of existence of the $V_{1,1}$ where periodic motions coexist with chaotic oscillations. Here, the system may chose a periodic motion or chaotic oscillations, depending on the initial conditions. As an example Figure 12 gives the result of plotting of the basins of attraction for the 


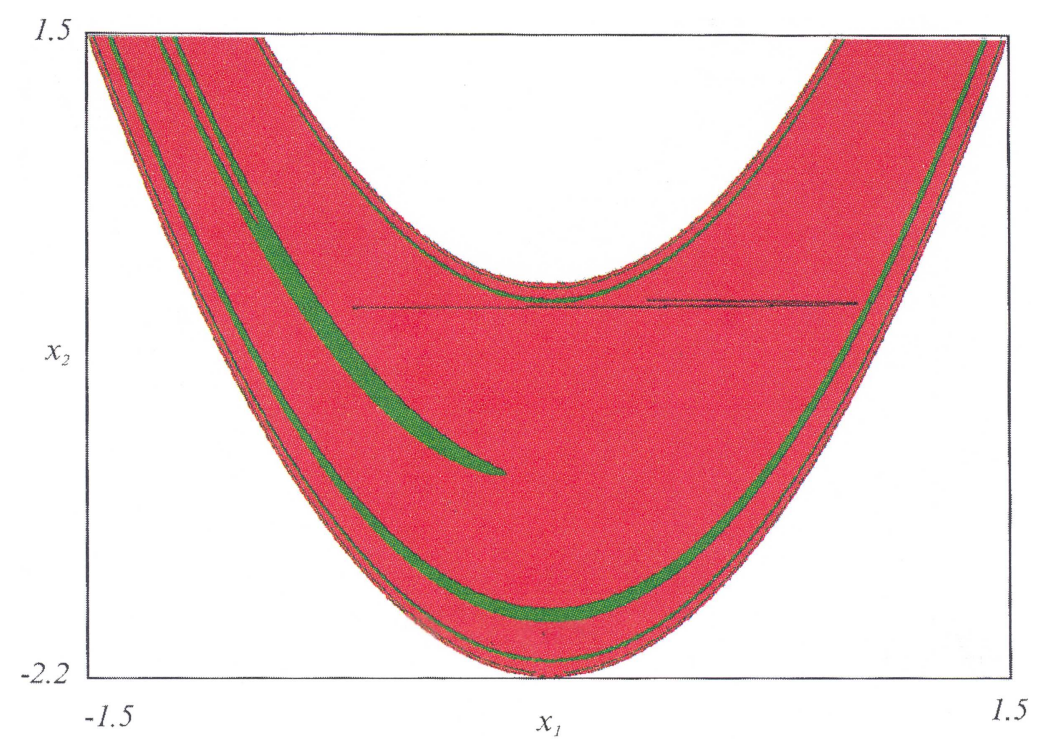

FIGURE 12 Basins of attraction for the coexisting 6-cycle and chaotic motion with $\alpha=1.7, \beta=-0.03$. (See Color Plate V.)

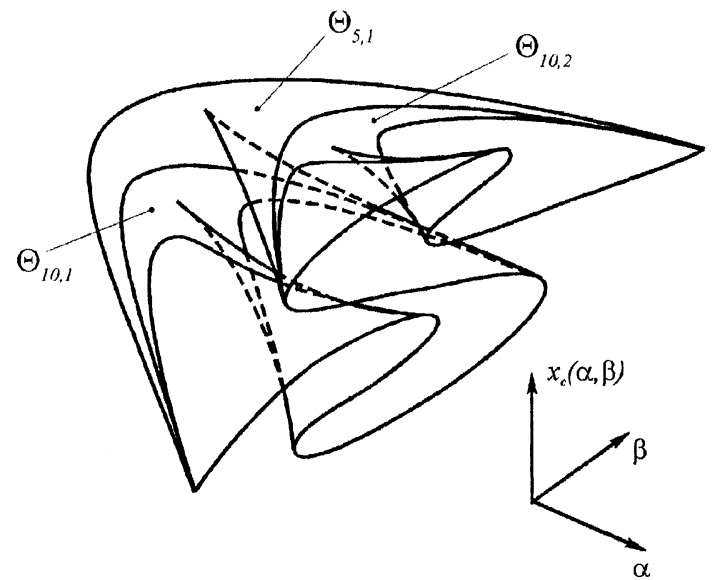

(a)

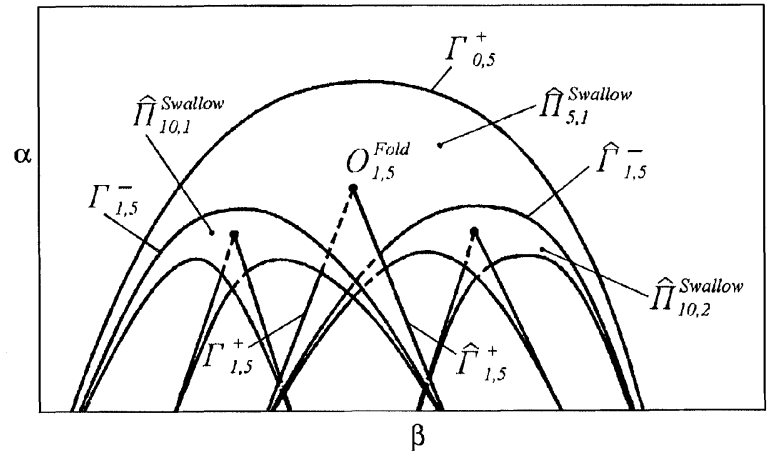

(b)

FIGURE 13 (a) The 2D surface illustrating the peculiarities of organization of the domains of existence for 5-cycle $\hat{\Pi}_{5,1}^{\text {Swallow }}$ and 10cycle $\hat{\Pi}_{10,1}^{\text {Swallow }}$ and $\hat{\Pi}_{10,2}^{\text {Swallow }}$ which are of a "swallow tail" kind. (b) Bifurcation curves bounding the domains $\hat{\Pi}_{5,1}^{\text {Swallow, }} \hat{\Pi}_{10,1}^{\text {Swallow }}$ and

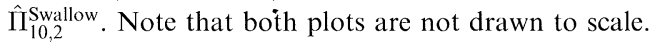


coexisting 6-cycle and chaotic motion $(P=$ $(1.7 ;-0.03))$.

Now let us consider the difference between the properties of the set $\Pi_{k}^{\text {Swallow }}$ and the set $\Pi_{k, j}$. Similarly to (Anishchenko, 1990), when analyzing the model of the radiophysical generator with inertial non-linearity, we introduce and consider a three-dimensional space $\left(\alpha, \beta, x_{c}(P)\right)$, in which we depict the dependence $x_{c}(P)$. Here, as in (Anishchenko, 1990), by $x_{c}(P)$ we mean one of component of the fixed point $X_{c}(P)$ of the mapping $X_{c}=F^{m}\left(X_{c}\right)$. The locus, corresponding to stable and unstable $m$-cycles, forms a twodimensional surface $\Theta_{m, j}$ in $\left(\alpha, \beta, x_{c}(P)\right)$. The first index in the designation of $\Theta_{m, j}$ indicates the value of $m$, for which the surface was built, and the second index is introduced to distinguish $\Theta_{m, j}$, having the same $m$. The plot of such a surface for $m=5$ and $m=10$ is schematically shown $\left(\Theta_{5,1}\right.$ and $\left.\Theta_{10,1}, \Theta_{10,2}\right)$ in Figure 13(a).

Now we shall project an image of the surface $\Theta_{m, j}$ onto the plane of control parameters (Fig. 13(b)). For the purpose of illustration, the bifurcation curves in Figure 13 are not drawn to scale. The domains of existence of the stable 5cycle and the stable 10-cycles are denoted as

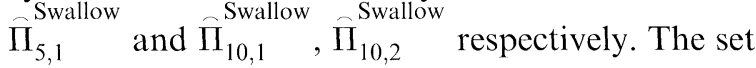
Swallow

$\Pi_{5,1}$ is limited by the period-doubling bifurcation curves $\Gamma_{1,5}^{-}, \widehat{\Gamma}_{1,5}$ and the curves $\Gamma_{0,5}^{+}, \Gamma_{1,5}^{+}, \widehat{\Gamma}_{1,5}^{+}$ $\left(\Gamma_{1,5}^{+}, \Gamma_{1,5}^{+}\right.$- are the lines of fold (Arnold, 1990) of $\left.\Theta_{5,1}\right)$, corresponding to combination of the stable 5-cycle and the unstable 5-cycle (the curve $N_{+}$). The curves $\Gamma_{1,5}^{+}$and $\widehat{\Gamma}_{1,5}^{+}$are supported by the bifurcation point $O_{1,5}^{\text {Fold }}\left(O_{1,5}^{\text {Fold }} \in \widehat{\Pi}_{5,1}^{\text {Swallow }}\right)$ of codimension 2 (the point of assembly (Arnold, 1990) of the projection $\Theta_{5,1}$ ), forming a sector in $\widehat{\Pi}_{5,1}^{\text {Swallow }}$, where two stable 5-cycles and two unstable 5cycles exist. One of the unstable 5-cycles occurs together with the stable 5-cycle on the boundary $\Gamma_{0,5}^{+}$.

The picture below the curves $\Gamma_{\text {Swallow }}^{--}$and $\widehat{\Gamma}_{1,5}$ repeats structure described for $\widehat{\Pi}_{5,1}^{\text {Swallow }}$ (Figs. 13, 14). The bifurcation curves $\Gamma_{j, 5 \cdot 2^{i-1}}^{-}$and $\widehat{\Gamma}_{j, 5 \cdot 2^{i-1}}^{-}$, $j=1, \ldots, 2^{i-1} ; i=1,2, \ldots$ accumulate, and there

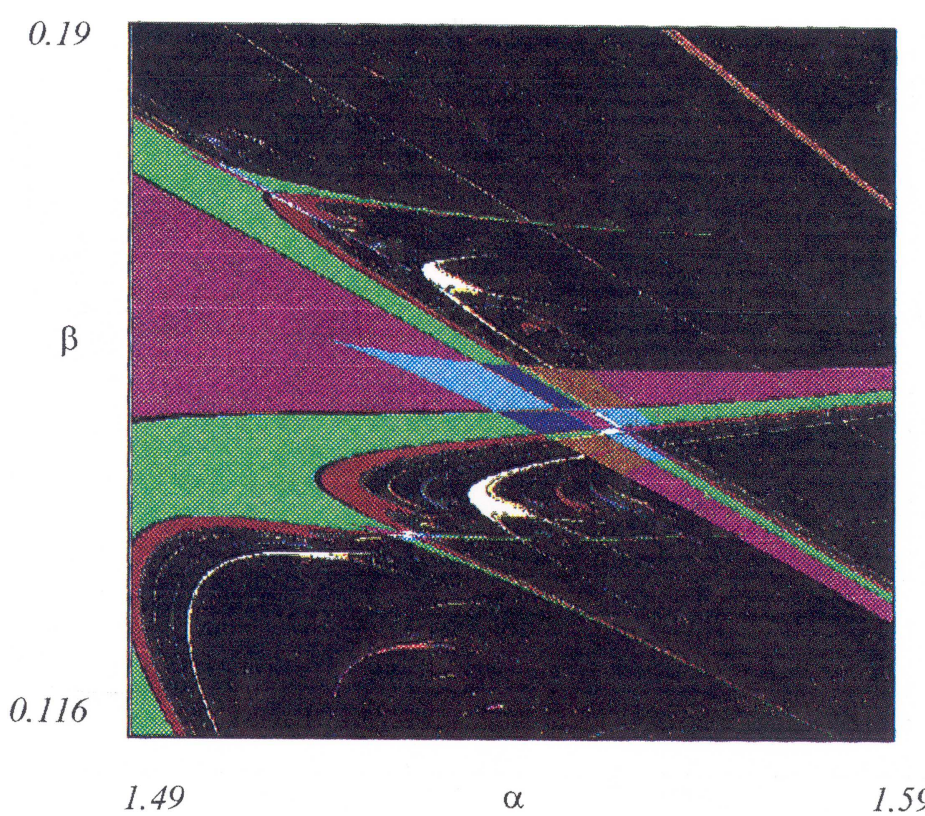

FIGURE 14 The results of numerical calculations of the domains $\Pi_{k}^{\text {Swallow }}, k=5,6,7,8$ and some others. (See Color Plate VI.) 


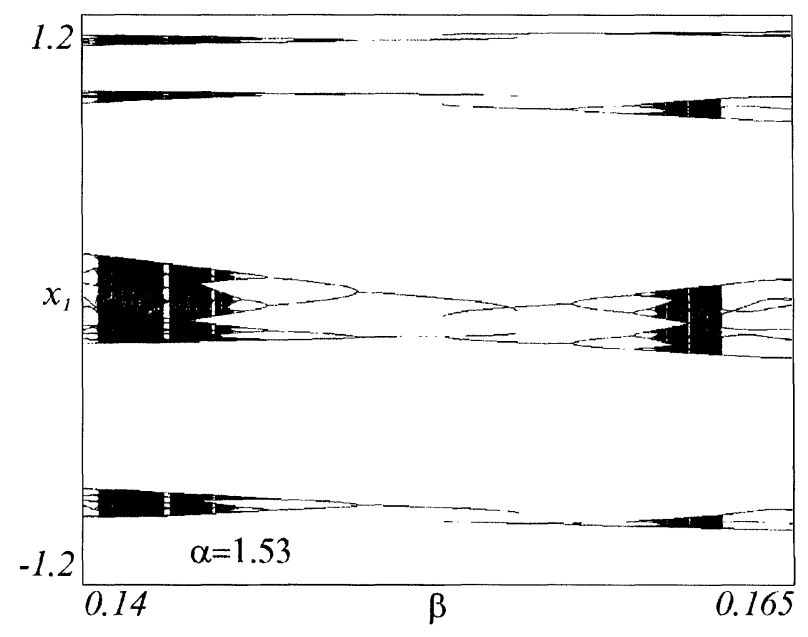

(a)

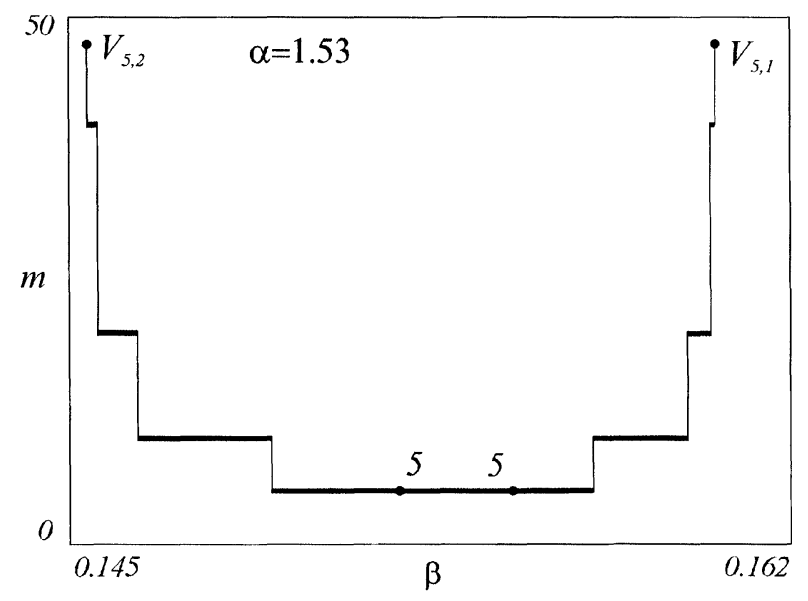

(b)

FIGURE 15 (a) Bifurcation diagram for $\alpha=1.53$ and $0.14 \leq \beta \leq 0.165$ and (b) branching pattern for $\alpha=1.53$ and $0.145 \leq \beta \leq 0.162$. This section intersects that region of the domain $\Pi_{5}^{\text {Swallow }}$ where two stable 5 -cycle coexists.

exist transversal directions along which infinite series of period-doubling bifurcations take place.

The projections of the two-dimensional surfaces $\Theta_{m, j}, \quad\left(m=5 \cdot 2^{i-1} ; \quad j=1, \ldots, 2^{i-1}, \quad i=2,3, \ldots\right)$ onto the place of control parameters have the same peculiarities as $\Theta_{5,1}$ (Fig. 14). The domain $\Pi_{5}^{\text {Swallow }}$ consists of the collection of the sets
$\widehat{\Pi}_{5 \cdot 2^{i-1}, j}^{\text {Swallow }}, j=1, \ldots, 2^{i-1} ; i=1,2 \ldots$ :

$$
\Pi_{5}^{\text {Swallow }}=\bigcup_{i=1}^{\infty}\left(\bigcup_{j=1}^{2^{i-1}} \widehat{\Pi}_{5 \cdot 2^{i-1}, j}^{\text {Swallow }}\right)
$$

Figure 14 (see also Figs. 4, 5) shows the

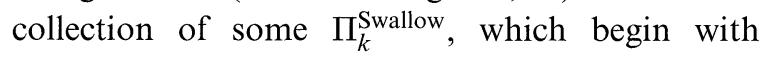




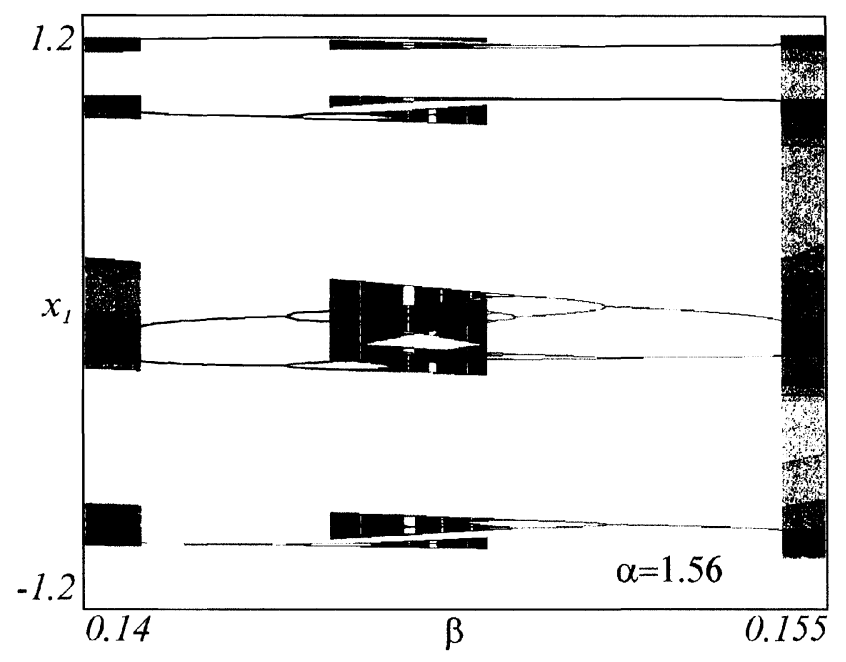

(a)

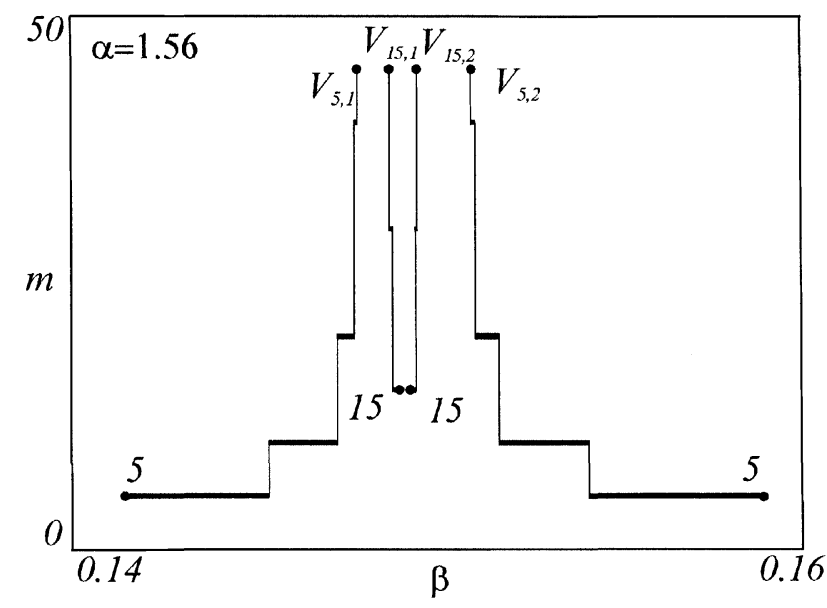

(b)

FIGURE 16 (a) Bifurcation diagram for $\alpha=1.56$ and $0.14 \leq \beta \leq 0.155$ and (b) branching pattern for $\alpha=1.56$ and $0.14 \leq \beta \leq 0.16$. This section intersects that region of the domain $\Pi_{5}^{\text {Swallow }}$ where two stable periodic, stable periodic and chaotic or two chaotic motions can coexist.

different stable $k$-cycles

$$
\Pi_{k}^{\text {Swallow }}=\bigcup_{i=1}^{\infty}\left(\bigcup_{j=1}^{2^{i-1}} \widehat{\Pi}_{k \cdot 2^{i-1}, j}^{\text {Swallow }}\right), \quad k=5,6,7,8 .
$$

Let us denote the part of $\Pi_{k}^{\text {Swallow }}$, where a single stable motion exists, which is uninterrupted along the parameters, as $\Pi_{k, \text { conv }}^{\text {Swallow }}$. The properties of $\Pi_{k, \text { conv }}^{\text {Swallow }}$ are similar to the above adduced properties of $\Pi_{k, j}$. There is non-uniqueness of motions in the domain $\Pi_{k}^{\text {Swallow }} \backslash \Pi_{k, \text { conv }}^{\text {Swallow }}$, which lies between the bifurcation curves $\Gamma_{j, 5 \cdot 2^{i-1}}^{+}$and $\widehat{\Gamma}_{j, 5 \cdot 2^{i-1}}^{+}, j=$ $1, \ldots, 2^{i-1} ; i=1,2, \ldots$ (Figs. 13,14 ).

When the boundaries of $\Pi_{k}^{\text {Swallow }} \backslash \Pi_{k, \text { conv }}^{\text {Swallow }}$ are intersected in the points of the curves $\Gamma_{j, 5 \cdot 2^{i-1}}^{+}$, and 
$\widehat{\Gamma}_{j, 5 \cdot 2^{i-1}}^{+} ; j=1, \ldots, 2^{i-1} ; i=1,2, \ldots$, one can observe hard transitions from one mode to another with hysteresis as it is typical for such transitions (Figs. 15 and 16). Figures 15 and 16 illustrate only one of possible variants of change in the character of the dynamics with variation in the parameters. The branching patterns in other sections may be more complicated in comparison with those shown in Figures 15(b) and 16(b) (see Figs. 17 and 18).
We realize that the content of the above analysis is qualitative rather than quantitative. As it is seen from Figure 14 the real picture of the properties of $\Pi_{k}^{\text {Swallow }}$ as a whole is substantially more complicated than presented in this paper.

Finally, the case should be considered, when the trajectory of deformation passes through $O_{j, 5 \cdot 2^{i-1}}^{\text {Fold }}$, $j=1, \ldots, 2^{i-1} ; i=1,2, \ldots$ The dependence of the solutions of $x_{c}(P)(2)$ on the parameters for this case is qualitatively shown in Figure 19, where

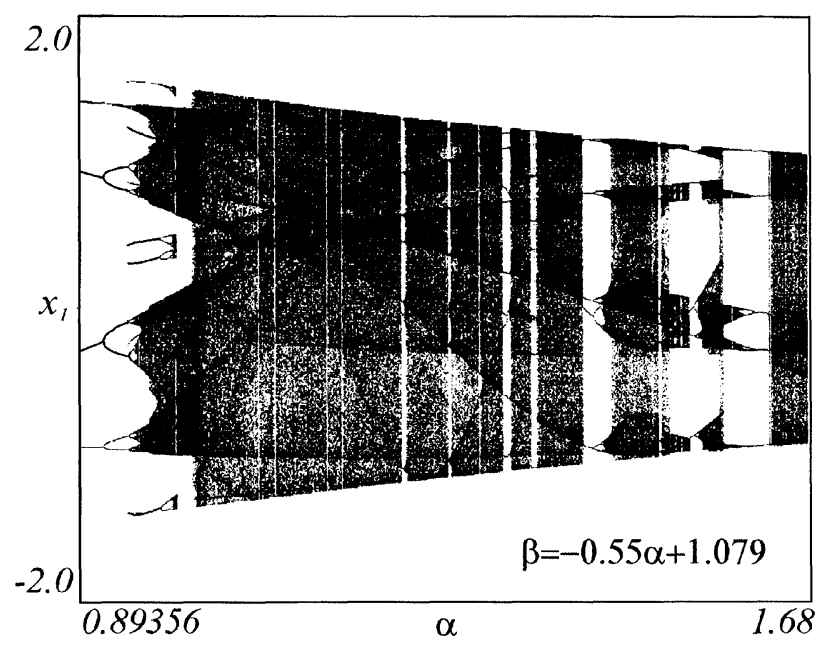

FIGURE 17 Bifurcation diagram for $\beta=-0.55 \alpha+1.079$ where $0.89356 \leq \alpha \leq 1.68$.

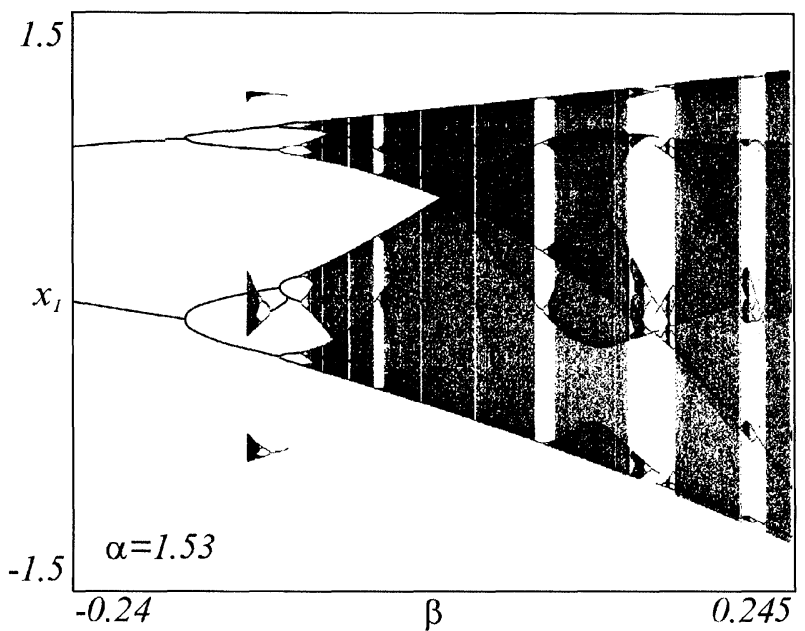

FIGURE 18 Bifurcation diagram for $\alpha=1.53$ and $-0.24 \leq \beta \leq 0.245$. 


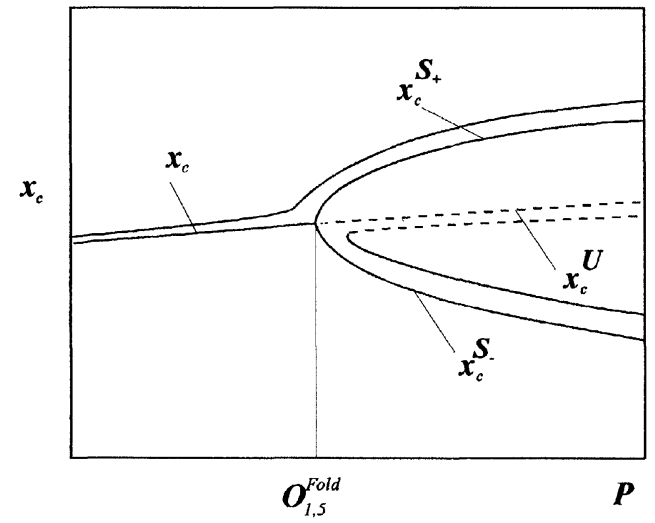

FIGURE 19 The qualitative plot of the variation of the stable and unstable 5-cycles with the parameters when the trajectory of deformation passes through the bifurcation point $O_{1,5}^{\text {Fold }}$ of codimension 2 .

$x_{c}^{S_{-}}(P)$ and $x_{c}^{S_{+}}(P)$ correspond to the stable 5cycles, and $x_{c}^{U}(P)$ corresponds to the unstable 5cycle. When approaching $O_{1,5}^{\text {Fold }}$ the solutions $x_{c}^{S_{-}}(P), x_{c}^{S_{+}}(P)$ and $x_{c}^{U}(P)$ become closer and then they combine in the point $O_{1,5}^{\text {Fold }}$ and form the single stable 5-cycle, which is dependent on $P$ uninterruptedly. The solution $x_{c}(P)$ is nonrough at the point $O_{1,5}^{\text {Fold }}$ and, if we change the trajectory of deformation in such a way that it does not pass through $O_{1,5}^{\text {Fold }}$, then $x_{c}(P)$ falls into two isolated solutions.

\section{CONCLUSIONS}

1. In the present paper we have established the domains of the modes of periodic and chaotic oscillations in the plane of parameters using numerical as well as analytical approaches. Two types of the domains of cycles stability: $\Pi_{k, j}, \Pi_{k}^{\text {Swallow }}, k=1,2, \ldots$ were determined. The structure of sets $\Pi_{k, j}, \Pi_{k}^{\text {Swallow }}$ and their properties in whole have been studied.

2. It was shown that the motions, determined on the sets $\Pi_{k, j}$, depend smoothly on the parameters. While moving along the parameters continuously within the limits of $\Pi_{k, j}$, the transition from some stable cycles to other cycles occurs softly through a sequence of direct and reverse period-doubling bifurcations, whereas in $\Pi_{k}^{\text {Swallow }}$ hysteretic transitions are possible.

3. While intersecting the boundaries of $\Pi_{k, j}$, which correspond to the parameters of hard occurrence of stable cycles, catastrophic transitions from one stable motion to another or catastrophic chaotization are possible. But such transitions are not hysteretic.

4. The sets $\Pi_{k, j}$ intersect non-emptily. Some of the regions $\Pi_{k, j}$ have intersections with the domains where chaotic oscillations are realized, and that is why a great variety of bifurcation transitions is possible.

\section{References}

Alligood, K. T. and Sauer, T. (1988) Rotation Numbers of Periodic Orbits in the Hénon Map. Comm. Math. Phys., 120, 105.

Anishchenko, V. (1990) Complex Oscillations in Simple Systems. Nauka, Moscow. (in Russian).

Arnold, V. (1990) Catastrophic Theory. Nauka, Moscow. (in Russian).

Barfred, M., Mosekilde, E. and Holstein-Rathlou, N.-H. (1996) Bifurcation Analysis of Nephron Pressure and Flow Regulation. Chaos, 6(3), 280-287.

Baushev, V., Zhusubaliyev, Zh. and Michal'chenko, S. (1996) Stochastic Features in the Dynamic Characteristics of a Pulse-Width Controlled Voltage Stabilizer. Electrical Technology, 1, 137-150. (Elsevier Science).

Baushev, V. and Zhusubaliyev, Zh. (1992) Indeterminable States of a Voltage Regulator with Pulse-Width Control. Electrical Technology, 3, 85-98 (Elsevier Science).

Bergé, P., Pomeau, Y. and Vidal, Ch. (1984) Order within Chaos (Towards a Deterministic Approach to Turbulence). John Wiley and Sons, New York.

Butenin, N., Neymark, Yu. and Fufayev, N. (1987) Introduction to the Theory of Nonlinear Oscillations. Nauka, Moscow. (in Russian).

Currey, J. (1979) On the Hénon Transformation. Comm. Math. Phys., 68, 129-140.

Derrida, B., Gervois, A. and Pomeau, Y. (1979) Universal Metric Properties of Bifurcations and Endomorphisms. J. Phys. A, 12, 269-296.

Dmitriev, A., Starkov, S. and Shirokov, M. (1994) The Structure of Periodic Orbits of Chaotic Self-Oscillating System Described by Difference Equations of the Second Order. Radiotechnika i Electronika, 8, 9, 1392-1400 (in Russian).

Gallas, J. (1994) Dissecting Shrimps: Results for Some OneDimensional Physical Models. Physica A, 202, 196-223.

Hansen, K. and Cvitanović, P. (1998) Bifurcation Structures in Maps of Hénon Type. Nonlinearity, 11, 1233-1261.

Hitzl, D. and Zele, F. (1981) An Exploration of the Hénon Attractors. J. Stat. Phys., 26(4), 683-695.

Hénon, M. (1976) A Two-Dimensional Mapping with a Strange Attractor. Comm. Math. Phys., 6(8), 69-77. 
Kan, I., Kocak, H. and Yorke, J. A. (1995) Persistent Homoclinic Tangencies in the Hénon Family. Physica D, 83, $313-325$.

Kuznetsov, A., Kuznetsov, S. and Sataev, I. (1993) Variety of Types of Critical Behavior and Multistability in PeriodDoubling Systems with Unidirectional Coupling Near the Onset of Chaos. Int. J. Bifurcation and Chaos, 3(1), 139-152.

Kuznetsov, A., Kuznetsov, S. and Sataev, I. (1994) From Bimodal One-Dimensional Maps to Hénon-like Two Dimensional Maps: Does Quantitative Universality Survive? Physics Letters A, 184, 413-421.

Landa, P. (1996) Nonlinear Oscillations and Waves in Dynamical Systems. Kluwer Academic Publ., Dordrecht-BostonLondon.

Mira, C., Carcasses, J., Millérioux, G. and Gardini, L. (1996) Plane Foliation of Two-Dimensional Noninvertible Maps. Int. J. Bifurcation and Chaos, 6(8), 1439-1462.

Moon, F. (1987) Chaotic Vibrations. An Introduction for Applied Scientists and Engineers, John Wiley and Sons, New York.
Mosekilde, E. (1996) Topics in Nonlinear Dynamics. Applications to Physics, Biology and Economic Systems, World Scientific.

Newhouse, S. (1974) Diffeomorphisms with Infinitely Many Sinks. Topology, 13, 9-18.

Neymark, Yu. and Landa, P. (1987) Stochastic and Chaotic Oscillations. Nauka, Moscow. (in Russian).

Schuster, H. (1984) Deterministic Chaos. Weinheim: PhysikVerlag.

Simo, C. (1979) On the Hénon-Pomeau Attractor. J. Stat. Phys., 21(4), 465-494.

Sonis, M. (1996) Once More on the Hénon Map: Analysis of Bifurcations. Chaos, Solitons and Fractals, 7(12), 2215-2234.

Zhusubaliyev, Zh. (1997) Bifurcations and Chaotic Motions in the Dynamics of the Relay Automatic Control Systems. In: Proc. of the 3rd International Conf. "Recognition-97”, Kursk, pp. 25-30 (in Russian).

Zhusubaliyev, Zh. (1997a) On Investigation of Chaotic Regimes of a Voltage Converter with Pulse-Width Modulation. Elektrichestvo, 6, 40-46 (in Russian). 


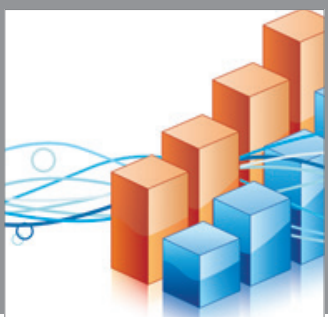

Advances in

Operations Research

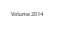

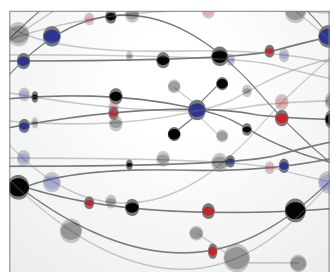

\section{The Scientific} World Journal
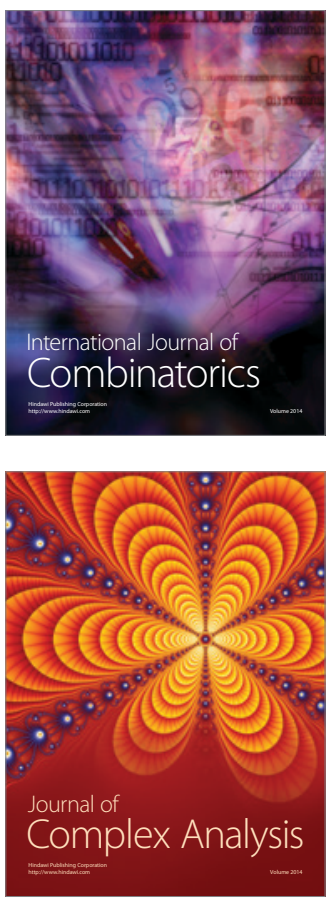

International Journal of

Mathematics and

Mathematical

Sciences
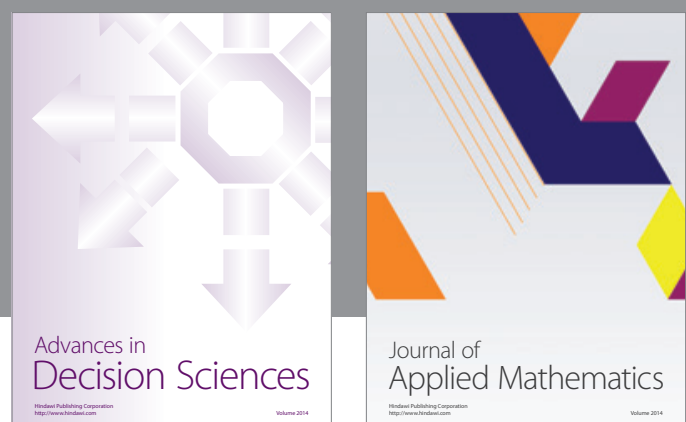

Journal of

Applied Mathematics
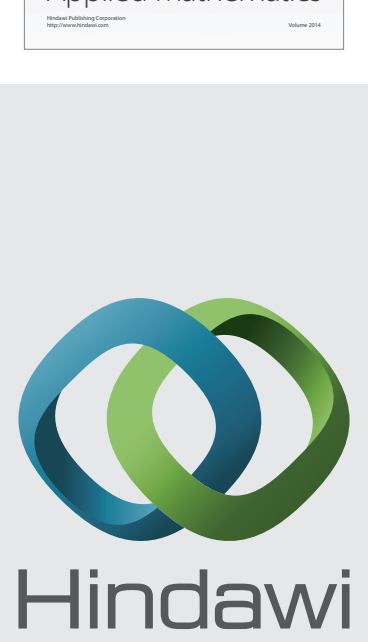

Submit your manuscripts at http://www.hindawi.com
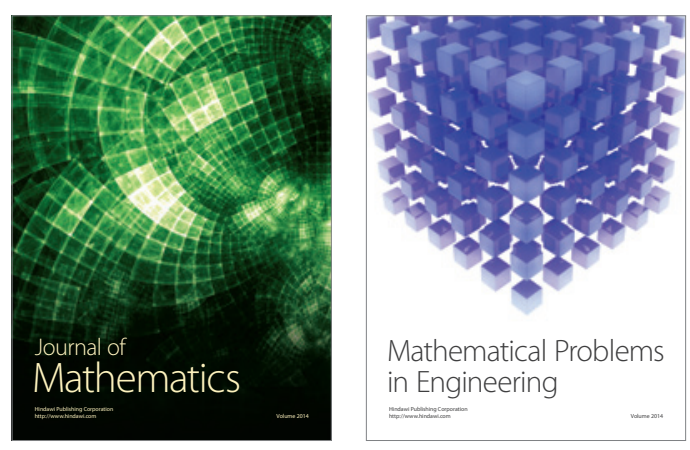

Mathematical Problems in Engineering
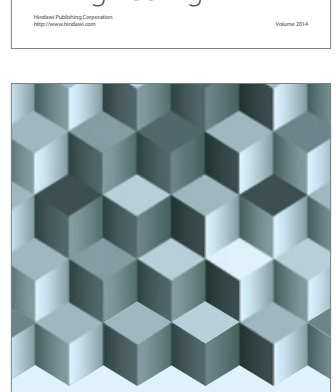

Journal of

Function Spaces
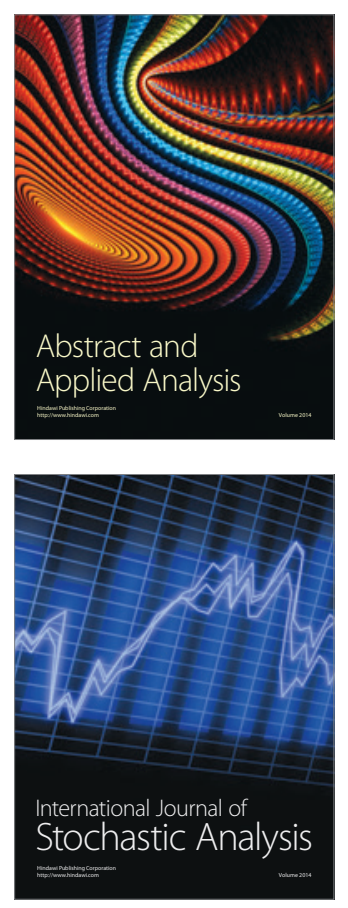

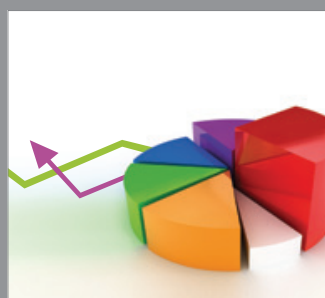

ournal of

Probability and Statistics

Promensencen
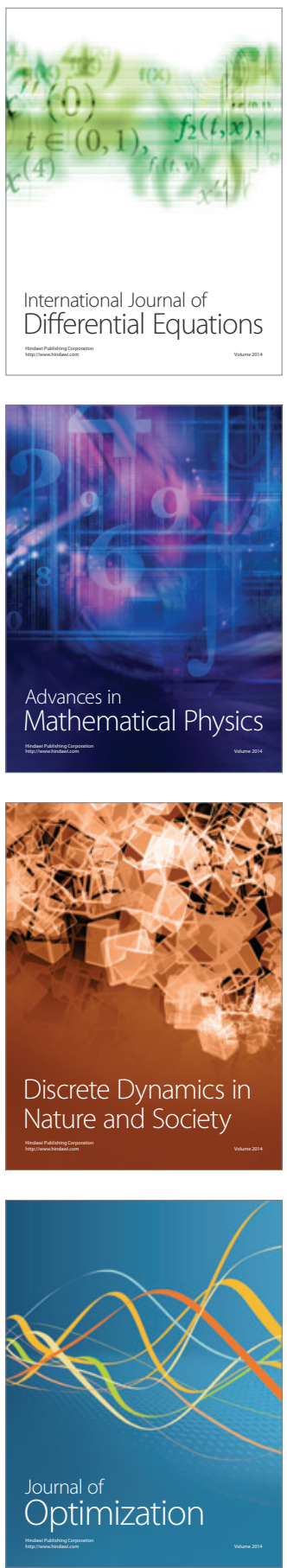